\title{
The Gamma-glutamyl Transpeptidase to Platelet Ratio (GPR) Predict Significant Liver Fibrosis and Cirrhosis in Chronic Egyptian HCV Patients
}

\author{
Ali Nada (MD) ${ }^{1}$, Aliaa Sabry (MD) ${ }^{1}$, Naglaa S. Elabd (MD) ${ }^{2}$, \\ Osama Elbahr (MD) ${ }^{1}$ \\ ${ }^{1}$ Hepatology and Gastroenterology, National Liver Institute, Menoufia university, Menoufia \\ Egypt \\ ${ }^{2}$ Department of Tropical Medicine, Faculty of Medicine, Menoufia University, \\ Menoufia, Egypt
}

Corresponding Author Naglaa S. Elabd, M.D.

\section{Mobile:}

00201092304322

\section{E mail:} naglaa_elabd@yahoo. com

Key words: Chronic HCV, Liver fibrosis, Non-invasive test
Background and aim: Non- invasive parameters of liver fibrosis are being widely incorporated and adopted in clinical practice, of them, 2 ratios APRI and FIB-4 were proposed and applied. The gamma-glutamyl transferase -toplatelet ratio (GPR) was developed and investigated as available test that is useful in predicting liver fibrosis stages in chronic HBV patients. We aimed to estimate the diagnostic performance of GPR compared to APRI in assessing different fibrosis stages estimated by ultrasound based Transient Elastography in chronic HCV Egyptian patients.

Methods: This prospective study was conducted on 90 treatment-naive chronic $\mathrm{HCV}$ patients. Fibrosis stages were assessed by Transient Elastography. Serum aspartate transferase, alanine transferase, gamma-glutamyl transferase and platelets were estimated. APRI and GPR formulas were calculated.
Results: GPR was significantly different with fibrosis stage ( $p$ value $<0.001)$ in addition, higher GPR values associated with increasing fibrosis stage. The mean \pm SD for GPR, APRI were $0.4 \pm 0.31$ and $0.38 \pm 0.34$ respectively. The AUROC of the GPR and APRI in predicting significant fibrosis (>F3) from nonsignificant fibrosis $(<\mathrm{F} 3)$ was significant with GPR AUC was 0.97 while APRI AUC was 0.86. GPR at cutoff point 0.31 has sensitivity $92 \%$, specificity $88 \%$, PPV $86 \%$ and NPV $91 \%$. While, APRI at cutoff 0.26 has sensitivity $81 \%$ and specificity $78 \%$.

Conclusion: The GPR represents a simple, non-invasive, inexpensive model for assessment of liver fibrosis. The GPR is a more accurate model than APRI to stage liver fibrosis in chronic $\mathrm{HCV}$ patients in Egypt.

\section{INTRODUCTION}

Chronic liver injury whatever the etiology leads to liver fibrosis. which results in structural and functional hepatic changes with extracellular matrix accumulation in diffuse pattern due to disturbed balance in their production, deposition, and degradation of fibrosis $[\mathbf{1 , 2}]$.

Different stages of fibrosis are important issues for diagnosis, follow up and treatment in patients with chronic liver disease. For decades, liver biopsy was believed to be the standard reference for evaluating the stage of fibrosis and degree of inflammation with development of different histological scoring systems $[3,4,5,6]$.

Although liver biopsy is considered the gold standard in assessment of liver fibrosis in addition to diagnosis of some conditions but its drawbacks as invasive procedure limit its use as well as sampling variations and patient's preference [7, 8, 9]. Hepatic fibrogenesis was originally considered irreversible condition, but it now considered as a dynamic process with potential resolution and irreversibility. 
single snapshot diagnosis of fibrosis by liver biopsy may not be representative of the continuous fibrosis process over time which further emphasize the need of repeated monitoring of fibrosis $[\mathbf{1 0}, \mathbf{1 1}, \mathbf{1 2}]$.

From all this points a question about the role of liver biopsy as the gold standard in estimating the diagnosis, severity and prognosis of fibrosis $[13,14]$. Non-invasive assessment tools of liver fibrosis have showed essential role in the previous few years with development of a wide spectrum of non-invasive tools starting from different direct and indirect markers to imagingbased techniques. These non-invasive parameters are widely used in clinical practice $[15,16,17,18]$, some have been validated in different studies, like transient elastography and the Fibrotest/ Fibrosure in Europe, especially in chronic HCVrelated cirrhosis [19]. Furthermore, no ideal model or test could replace liver biopsy completely which still the main tool to diagnose some complex hepatic problems in chronic liver disease [20].

Aspartate aminotransferase-to-Platelet ratio index (APRI) and fibrosis index based on the 4 factors (FIB-4) are 2 ratio models that have proposed and applied as indirect markers of liver fibrosis [21, 22].

Lemoine and his colleagues have evolved gamma-glutamyl transferase -to- platelet ratio (GPR) model, as helpful test that is valuable in chronic hepatitis B (CHB) patients in West Africa for estimation of the level of fibrosis in liver tissue [23]. Gamma-glutamyl transferase is a membrane-bound enzyme that is essential for synthesis of glutathione and has been considered a biomarker of hepatocellular damage [24].

In our study, we aimed to assess the diagnostic performance of GPR compared to APRI for estimation of different stages of fibrosis estimated by ultrasound based Transient Elastography (TE) in chronic HCV Egyptian patients.

\section{MATERIALS AND METHODS}

\section{Study population:}

This prospective research was performed on ninety participants. They were treatment-naive chronic $\mathrm{HCV}$ patients recruited from $\mathrm{HCV}$ treatment clinic at National Liver Institute (NLI) and Tropical Medicine department, faculty of
Medicine, Menoufia University in the period between August 2018 and January 2020.

Patients with the following criteria were included in the study: age more than 18 years, +ve HCV antibodies that were confirmed by PCR for HCV-RNA and all are naïve to treatment. Patients with previous HCV treatment or HIV coinfection in addition to patients with chronic conditions other than hepatitis $\mathrm{C}$ that could lead to liver inflammation and fibrosis such as, autoimmune hepatitis, other chronic viral infections as chronic HBV \& HDV, cholestatic or metabolic liver diseases, hepatocellular carcinoma (HCC) patients and patients with chronic alcohol consumption were excluded from this study. Our research was executed in correspondence with the Declaration of Helsinki. All participants provided an informed consent before being included in the study.

For all patients, history taking and clinical evaluation were done. Liver function tests [aspartate transaminase (AST), alanine transaminase (ALT), gamma-glutamyl transferase (GGT), serum total and direct bilirubin, serum albumin, prothrombin time and international normalized ratio (INR)], and complete blood count were assessed in addition to imaging studies including abdominal ultrasound and Transient Elastography.

\section{Assessment of fibrosis stages:}

Fibrosis stages were assessed by performing Transient Elastography (TE) (FibroscanR, EchosensR, Paris, France). Results in Kilopascals (Kpa) were expressed and corresponded to the median value of ten standardized measurements. A success rate of at least 60 percent of the total number of acquisitions with interquartile range lower than 30 percent of the median value, were considered reliable [25].

Patients with suspected liver inflammation (those with elevated hepatic transaminases $>2$ folds from the upper limit of normal) [26,27], those with body mass index more than 28 as well as postprandial measurements were excluded to avoid unreliability of the results [28].

\section{Laboratory assays:}

For platelet assessment, a Sysmex-XT4000i automated haematology analyzer and auxiliary reagents with normal range of platelet count $(125-350) \times 10^{9} / \mathrm{L}$. For evaluation of liver enzymes (aspartate transferase, alanine 
transferase, and gamma-glutamyl transferase) a Hitachi 7600 automated biochemistry analyzer and auxiliary reagents were used, with normal ranges were (15-40) IU/L, (9-50) IU/L, and (1060) IU/L respectively.

The third generation enzyme immunoassay (Ortho HCV version 3.0 ELISA; Ortho- Clinical Diagnostics INC., Raritan. NJ, USA) was used for detection of Anti-HCV antibody. HCV RNA level was measured quantitatively by means of Abbott Real Time HCV RNA Assay with lower detection limit $15 \mathrm{IU} / \mathrm{ml}$.

\section{Models calculation:}

The formulas for GPR, APRI, are as follows:

- $\mathrm{GPR}=\frac{\mathrm{GGT}(/ \mathrm{ULN})}{\mathrm{PLT} \text { counts }\left(10 \mathrm{x}^{9} / L\right)} \times 100 \quad[23]$

- $\mathrm{APRI}=\frac{\mathrm{AST}(/ \mathrm{ULN})}{\mathrm{PLT} \operatorname{counts}\left(10 \mathrm{x}^{9} / L\right)} \times 100[21]$

(ULN $=$ upper limit of normal for that laboratory).

\section{Statistical analysis:}

The baseline characteristics of patients are presented as follows: normal distribution data as mean \pm standard deviation, non-normal distribution continuous data as median (interquartile range (IQR), and categorical variables as number (percentage). Chi-square test (for categorical variables), Mann-Whitney test (for non-normal distribution continuous variables), and $\mathrm{t}$ test (for normal distribution variables).

Receiver operating characteristic (ROC) curve and the area under the ROC curve (AUROC) were used to evaluate the diagnostic performance of GPR versus APRI in predicting the different levels of liver fibrosis. All statistical analyses were carried out using the SPSS statistical software version 15.0 (SPSS Inc., Chicago, IL).

\section{RESULTS}

The baseline characteristics of the patients included in our study were presented in Table $\mathbf{1}$. The majority of patients were men $(66.7 \%)$, females represented $33.3 \%$, with mean \pm SD for age was $48.18 \pm 9.8$ years. The fibrosis stages measured by Fibroscan were distributed as follow, F0 in 18 patients (20\%), F0-F1 in 4 cases $(4.4 \%), \mathrm{F} 1-\mathrm{F} 2$ in 10 patients $(11.1 \%), \mathrm{F} 2$ in 2 patients (2.2\%), F3 in 9 cases (10\%), F3-4 in 7 patients (7.8\%) and $\mathrm{F} 4$ was present in 26 patients $(28.9 \%)$. The mean \pm SD for GPR, APRI were $0.4 \pm 0.31$ and $0.38 \pm 0.34$ respectively.

Table 2 represented the distribution of different fibrosis stages in relation to the age of the patients, GPR and APRI. We found that mean \pm SD of different stages of fibrosis was statistically varied with different age groups ( $p$ value was $<0.001$ ). In addition, GPR and APRI were significantly different with fibrosis stage ( $p$ value was < 0.001). Furthermore, we observed higher GPR and APRI with increasing fibrosis stage.

Receiver operating characteristic curve (ROC curve) analysis as a diagnostic performance in differentiating between significant $(>$ F3) and non-significant $(<\mathrm{F} 3)$ fibrosis revealed that The AUROC of the GPR and APRI in predicting significant fibrosis were significantly larger than the area under the diagonal reference line as presented in table 3 and figure 1

GPR at a cutoff point 0.31 has sensitivity $92 \%$ and specificity $88 \%$ with PPV and NPV $86 \%$ and $91 \%$ respectively. However, APRI at a cutoff point 0.26 has sensitivity $81 \%$ and specificity $78 \%$ with PPV $75 \%$ and NPV $82 \%$ (table 4)

Table (1): Baseline Characteristics of the Study Population.

\begin{tabular}{|l|l|l|}
\hline Characteristics & & no $(\%)$ \\
\hline Age, years (mean \pm SD) & & $48.18 \pm 9.8$ \\
\hline Fibroscan staging (Kp) & F0 & $18(20 \%)$ \\
& F0-F1 & $4(4.4 \%)$ \\
& F1 & $14(15.6)$ \\
& F1-F2 & $10(11.1 \%)$ \\
& F2 & $2(2.2 \%)$ \\
& F3 & $9(10 \%)$ \\
& F3-F4 & $7(7.8 \%)$ \\
& F4 & $26(28.9 \%)$ \\
\hline GPR (mean \pm SD) & & $0.4 \pm 0.31$ \\
\hline APRI (mean \pm SD) & & $0.38 \pm 0.34$ \\
\hline
\end{tabular}

GPR; gamma-glutamyl transferase -to- platelet ratio, APRI; Aspartate aminotransferase-to-Platelet ratio index

Nada et al., Afro-Egypt J Infect Endem Dis 2020;10(2):226-232

https://aeji.journals.ekb.eg/

http://mis.zu.edu.eg/ajied/home.aspx 
Table (2): Distribution of different fibrosis stages in relation to the age of the patients, GPR and APRI.

\begin{tabular}{|l|c|c|c|c|c|c|c|c|c|c|}
\hline $\begin{array}{l}(\mathbf{m e a n} \\
\mathbf{\pm S D})\end{array}$ & $\begin{array}{c}\text { F0 } \\
(\mathbf{n}=\mathbf{1 8})\end{array}$ & $\begin{array}{c}\text { F0-F1 } \\
(\mathbf{n}=\mathbf{4})\end{array}$ & $\begin{array}{c}\mathbf{F 1} \\
(\mathbf{n = 1 4})\end{array}$ & $\begin{array}{c}\mathbf{F 1 - F 2} \\
(\mathbf{n = 1 0})\end{array}$ & $\begin{array}{c}\text { F2 } \\
(\mathbf{n = 2})\end{array}$ & $\begin{array}{c}\text { F3 } \\
(\mathbf{n = 9})\end{array}$ & $\begin{array}{c}\text { F3-F4 } \\
(\mathbf{n = 7})\end{array}$ & $\begin{array}{c}\text { F4 } \\
(\mathbf{n = 2 6})\end{array}$ & ANOVA & P value \\
\hline Age & $42.2 \pm 9.3$ & $38.7 \pm 14.8$ & $47.7 \pm 9.4$ & $45.5 \pm 4.8$ & $42.5 \pm 20.5$ & $52.6 \pm 9$ & $49.5 \pm 6.1$ & $53.5 \pm 8.1$ & 3.7 & $<0.001$ \\
\hline GPR & $0.15 \pm 0.09$ & $0.26 \pm 0.04$ & $0.21 \pm 0.1$ & $0.21 \pm 0.05$ & $0.3 \pm 0.03$ & $0.64 \pm 0.27$ & $0.43 \pm 0.12$ & $0.68 \pm 0.34$ & 13.4 & $<0.001$ \\
\hline APRI & $0.16 \pm 0.07$ & $0.13 \pm 0.02$ & $0.26 \pm 0.1$ & $0.26 \pm 0.1$ & $0.24 \pm 0.11$ & $0.54 \pm 0.43$ & $0.42 \pm 0.4$ & $0.64 \pm 0.4$ & 5.7 & $<0.001$ \\
\hline
\end{tabular}

GPR; gamma-glutamyl transferase -to- platelet ratio, APRI; Aspartate aminotransferase-to-Platelet ratio index

Table (3): Area Under the curve (AUROC) of GPR and APRI to differentiate between nonsignificant $(<\mathrm{F} 3) \&$ significant $(>$ F3) fibrosis.

\begin{tabular}{|l|c|c|c|c|c|}
\hline Test Result Variable (s) & $\begin{array}{c}\text { Area } \\
\text { Under the } \\
\text { curve }\end{array}$ & Std. Error $^{\text {a }}$ & & Asymptotic Sig. & \multicolumn{2}{|c|}{$\begin{array}{c}\text { Asymptotic 95\% Confidence } \\
\text { Interval }\end{array}$} \\
\cline { 5 - 6 } & & & & Lower Bound & Upper Bound \\
\hline GPR & .970 & .014 & .000 & .942 & .997 \\
\hline APRI & .865 & .038 & .000 & .791 & .939 \\
\hline
\end{tabular}

GPR; gamma-glutamyl transferase -to- platelet ratio, APRI; Aspartate aminotransferase-to-Platelet ratio index

Table (4): Optimal cut-offs of GPR and APRI in predicting significant fibrosis.

\begin{tabular}{|l|c|c|c|c|c|}
\hline & Cut-off value & Sensitivity (\%) & Specificity (\%) & PPV (\%) & NPV (\%) \\
\hline GPR & 0.31 & 92 & 88 & 86 & 91 \\
\hline APRI & 0.26 & 81 & 78 & 75 & 82 \\
\hline
\end{tabular}

GPR; gamma-glutamyl transferase -to- platelet ratio, APRI; Aspartate aminotransferase-to-Platelet ratio index, PPV; positive predictive value, NPV; Negative predictive value.

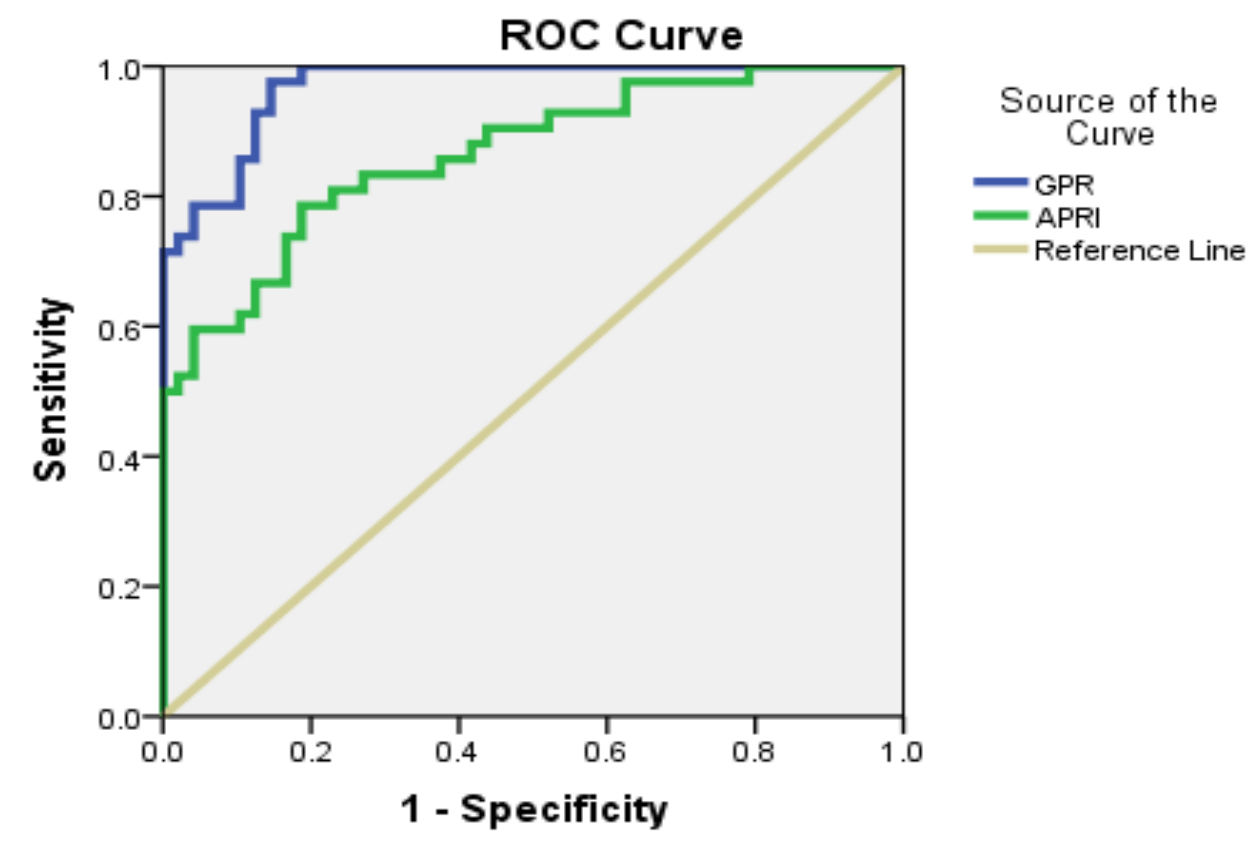

Figure 1: The ROC curves of APRI and GPR in differentiating between non-significant $(<\mathrm{F} 3)$ \& significant (> F3) fibrosis 


\section{DISCUSSION}

Chronic liver injury leads to hepatic fibrosis [1]. Non-invasive tools for assessment of fibrosis as different serum direct and indirect markers in addition to imaging- based techniques were utilized. Those non- invasive tools are frequently used in clinical practice [15].

Lemoine and his colleagues have developed a model of gamma-glutamyl transpeptidase-toplatelet ratio (GPR), as an available test that could be useful in predicting the levels of liver fibrosis in patients with chronic hepatitis B (CHB) in West Africa. [23] In our research, we aimed to assess the diagnostic performance of that ratio (GPR) in comparison with the APRI score for estimation of different stages of fibrosis estimated by ultrasound based Transient Elastography (TE) in chronic HCV Egyptian patients.

In our study enrollment occurred to chronic HCV patients who were candidate for antiviral treatment for assessment of severity of fibrosis before starting antiviral treatment using transient elastography (fibroscan). In spite of its high diagnostic performance, the Fibroscan device is still expensive and requires annual maintenance. In addition, in Egypt the machine is only available in few main hospitals all over the country, so there is a need to simple, precise, inexpensive and non-invasive test to assess the severity of fibrosis in comparison to fibroscan.

We staged the chronic HCV patients using the fibroscan from F0 to F4. When we applied GPR score in different fibrosis stages, we observed that GPR was significantly different with varied stages of fibrosis and the $\mathrm{p}$ value was $<0.001$. These results matched with Vardar et al who reported that GPR is suggested as a potential biomarker of fibrosis in a Turkish cohort [29]. Furthermore, we observed higher GPR with increasing the fibrosis stage its values were 0.64 $\pm 0.27,0.43 \pm 0.12$ and $0.68 \pm 0.34$ in F3, F3-4 and $\mathrm{F} 4$ respectively, however lower values were detected in F0, F0-1, F1, F1-2, F2 they were 0.15 $\pm 0.09,0.26 \pm 0.04,0.21 \pm 0.1,0.21 \pm 0.05$ and $0.3 \pm 0.03$ respectively. It was previously reported by Lemoine et al. [23] who developed the novel model of GPR that this model was valuable in predicting the levels of liver fibrosis in CHB patients in West Africa.

A preliminary investigation has pointed out that the diagnostic performance of gamma-glutamyl transpeptidase-to- platelet ratio (GPR) in predicting significant fibrosis $(\geq F 2)$, extensive fibrosis $(\geq F$ 3) and cirrhosis $(\geq F 4)$ was near to or higher than those of FIB-4 and APRI scores utilizing the METAVIR pathological scoring system as a reference [23]. Similarly, Bedossa et al. [30] has concluded that GPR was a significant test in detection and grading of hepatic fibrosis. These results were also confirmed by Zeng and his colleagues [31] when studying the performance of different easy, simple and noninvasive models for the assessment of significant liver fibrosis in chronic hepatitis B patients. They reported that GPR is deemed as a considerable test.

In the present study, the diagnostic performance of APRI and GPR in differentiating between non-significant $(<$ F3) and significant $(>$ F3) fibrosis by ROC curve analysis revealed that AUROC of the GPR and APRI in predicting significant fibrosis were significantly larger than the area under the diagonal reference line. The AUC of the GPR was 0.97 while that of APRI was 0.865 , which means that GPR is a valuable test to differentiate between significant and nonsignificant hepatic fibrosis where GPR at a cutoff point 0.31 has sensitivity $92 \%$ and specificity 88\% with PPV and NPV $86 \%$ and $91 \%$ respectively, and APRI at a cutoff point 0.26 has sensitivity $81 \%$ and specificity $78 \%$ with PPV $75 \%$ and NPV $82 \%$. These finding indicate that GPR performance in predicting different stages of liver fibrosis is better than those of APRI.

Previous reports on chronic HBV supported our findings, Where Lemoine et al. [23] in his cohort study has proposed that cutoff points of GPR $>0.32$ and $>0.56$ could be utilized for prediction of significant fibrosis and cirrhosis, respectively. In a study conducted by Liu and his colleagues [32] on $\mathrm{HBeAg}$-positive and HBeAg-negative patients, they reported that utilizing the cut-offs of GPR by Lemoine et al, in patients with $\mathrm{HBeAg}$-positive and $\mathrm{HBeAg}$ negative chronic liver disease the sensitivity and specificity in predicting significant fibrosis were $74.4 \%$ and $66.6 \%, 73.2 \%$ and $65.3 \%$, respectively. They reported that regarding the role of APRI, FIB-4 and GPR in prediction of different stages of liver fibrosis, all have moderate accuracy with GPR showed the best performance. 


\section{CONCLUSION}

Results demonstrated the remarkable role of GPR as a simple, accurate, non-invasive and inexpensive alternative test to Fibroscan for assessment of liver fibrosis in resourceconstrained countries like Egypt, in addition GPR performance is better than APRI scoring system. These findings might encourage the future use of GPR in evaluating liver fibrosis.

Funding: No funding resources

Conflict of Interest: the authors declare that there was no conflict of interest.

\section{Ethical consideration}

Our study was executed in correspondence with the Declaration of Helsinki. An informed consent was provided by all participants before entering the study, and, the ethics Committee of national liver institute, Menoufia University confirmed our research protocol.

\section{Highlights}

- Chronic liver injury whatever the etiology leads to liver fibrosis. Different stages of fibrosis are important issues for diagnosis.

- Although the liver biopsy is considered the gold standard in assessment of liver fibrosis but its drawbacks as invasive procedure limits its use.

- Gamma-glutamyl transferase -to- platelet ratio (GPR) model, as helpful test that is valuable in chronic hepatitis $\mathrm{B}(\mathrm{CHB})$

- We observed that GPR was significantly different with fibrosis stage.

- GPR represents a simple, non-invasive, inexpensive model for assessment of liver fibrosis.

\section{REFERENCES}

1. Yano M, Kumada H, Kage M, Ikeda K, Shimamatsu $\mathrm{K}$, Inoue $\mathrm{O}$, et al. The long-term pathological evolution of chronic hepatitis C. Hepatology 1996; 23:1334-1340.

2. Younossi ZM, Stepanova M, Rafiq N, Makhlouf H, Younoszai Z, Agrawal R, et al. Pathologic criteria for nonalcoholic steatohepatitis: interprotocol agreement and ability to predict liver-related mortality. Hepatology 2011; 53:1874-1882.
3. Knodell RG, Ishak KG, Black WC. "Formulation and application of a numerical scoring system for assessing histological activity in asymptomatic chronic active hepatitis," Hepatology 1981; 1(5): 431-435.

4. Standish RA, Cholongitas E, Dhillon A, Burroughs AK, Dhillon AP. An appraisal of the histopathological assessment of liver fibrosis. Gut 2006; 55(4): 569-578.

5. Scheuer PJ. Classification of chronic viral hepatitis: a need for reassessment. Journal of Hepatology 1991; 13 (3) 372-374.

6. Sebastiani G, Alberti A. Non-invasive fibrosis biomarkers reduce but not substitute the need for liver biopsy. World Journal of Gastroenterology 2006; 12(23): 3682-3694.

7. Cadranel JF, Rufat P. Degos F. Practice of liver biopsy in France: results of a prospective nationwide survey. For the Group of epidemiology of the French Association for the study of the liver (AEF). Hepatology 2000; 32: 477-481

8. Bravo AA, Sheth SG, Chopra S. Liver biopsy. $N$ Engl J Med 2001; 344:495-500.

9. Piccinino F, Sagnelli E, Pasquale G, Giusti G. Complications following percutaneous liver biopsy. A multicenter retrospective study on 68,276 biopsies. J Hepatol 1986; 2:165-173.

10. Regev A, Berho M, Jeffers LJ, Milikowski C, Molina EG, Pyrsopoulos NT, et al. Sampling error and intraobserver variation in liver biopsy in patients with chronic $\mathrm{HCV}$ infection. Am J Gastroenterol 2002; 97:2614-2618.

11. Chang TT, Liaw YF, Wu SS, et al. Long-term entecavir therapy results in the reversal of fibrosis/cirrhosis and continued histological improvement in patients with chronic hepatitis B. Hepatology 2010;52:886-893.

12. George SL, Baon BR, Brunt EM, et al. Clinical, virologic, histologic, and biochemical outcomes after successful HCV therapy: a 5-year followup of 150 patients. Hepatology 2009; 49:729738.

13. Standish RA, Cholongitas E, Dhillon A, Burroughs AK, Dhillon AP. An appraisal of the histopathological assessment of liver fibrosis. Gut 2006; 55:569-578. doi:10.1136/gut. 2005.084475

14. Bedossa P, and Carrat F. Liver biopsy: the best, not the gold standard. J.Hepatol 2009; 50;(1) 3 . doi:10.1016/j. jhep.2008.10.014. 
15. Castera L. Invasive and non-invasive methods for the assessment of fibrosis and disease progression in chronic liver disease," Best Practice and Research: Clinical Gastroenterology 2011; 25(2):291-303.

16. Parkes J, Guha IN, Roderick P, Rosenberg W. Performance of serum marker panels for liver fibrosis in chronic hepatitis C," Journal of Hepatology 2006; 44(3): 462-474.

17. Pinzani M, Vizzutti F, Arena U, Marra F. Technology Insight: noninvasive assessment of liver fibrosis by biochemical scores and elastography," Nature Clinical Practice Gastroenterology \& Hepatology 2008; 5(2):95106.

18. Chi HH, Verveer C, Hansen BE, Zondervan PE, Janssen HLA, de Knegt RJ. Exclusion of the percutaneous liver biopsy from the management of chronic hepatitis $\mathrm{B}$ and $\mathrm{C}$ patients: are essential secondary diagnosis being missed?" Journal of Hepatology 2012; 56(2), 411-412.

19. Vasilios Papastergiou, Emmanuel Tsochatzis, Andrew K. Burroughs. Non-invasive assessment of liver fibrosis. Annals of Gastroenterology 2012; 25:218-231.

20. EASL-ALEH Clinical Practice Guidelines: Noninvasive tests for evaluation of liver disease severity and prognosis. Journal of Hepatology 2015; 63:237-264.

21. Wai CT, Greenson JK, Fontana RJ, Kalbfleisch JD, Marrero JA, Conjeevaram HS et al. A simple noninvasive index can predict both significant fibrosis and cirrhosis in patients with chronic hepatitis C. Hepatology 2003; 38(20):518-526.

22. Xiao G, Yang J, Yan L. Comparison of diagnostic accuracy of aspartate aminotransferase to platelet ratio index and fibrosis-4 index for detecting liver fibrosis in adult patients with chronic hepatitis $\mathrm{B}$ virus infection: a systemic review and meta-analysis. Hepatology 2015; 61:292-302.

23. Lemoine M, Shimakawa $Y$, Nayagam S, Khalil M, Suso P, Lloyd J et al. The gamma-glutamyl transpeptidase to platelet ratio (GPR) predicts significant liver fibrosis and cirrhosis in patients with chronic HBV infection in West Africa. Gut 2016; 65(8):1369-1376.
24. Whitfield JB. Gamma glutamyl transferase. Critical Reviews in Clinical Laboratory Sciences 2001; 38(4):263-355. doi: $10.1080 / 20014091084227$.

[PubMed]

[CrossRef] [Google Scholar]

25. Castera L, Forns X, Alberti A. Non-invasive evaluation of liver fibrosis using transient elastography. J Hepatol 2008; 48(5):835-847.

26. Coco B, Oliveri F, Maina AM, Ciccorossi P, Sacco R, Colombatto P, et al. Transient elastography: a new surrogate marker of liver fibrosis influenced by major changes of transaminases. J Viral Hepat 2007; 14(5):360369.

27. Arena U, Vizzutti F, Corti G, Ambu S, Stasi C, Bresci S, et al. Acute viral hepatitis increases liver stiffness values measured by transient elastography. Hepatology 2008;47(2)380-384.

28. Tsochatzis EA, Gurusamy KS, Ntaoula S, Cholongitas E, Davidson BR, Burroughs AK, et al. Elastography for the diagnosis of severity of fibrosis in chronic liver disease: a meta-analysis of diagnostic accuracy. J Hepatol 2011; 54(4):650-659.

29. Vardar R, Vardar E, Demiri S, Sayhan SE, Bayol U, Yildiz C, et al. Is there any noninvasive marker replace the needle liver biopsy predictive for liver fibrosis, in patients with chronic hepatitis? Hepatogastroenterology 2009; 56:1459-65.

30. Bedossa P, Poynard T. An algorithm for the grading of activity in chronic hepatitis $\mathrm{C}$. The METAVIR Cooperative Study Group. Hepatology 1996; 24(2):289-293.

31. Zeng X, Xu C, He D, Maoshi Li, Huiyan Zhang, Quanxin $\mathrm{Wu}$, et al. Performance of several simple, noninvasive models for assessing significant liver fibrosis in patients with chronic hepatitis B. Croat Med J 2015; 56(3):272-279.

32. Liu DP, Lu W, Zhang ZQ, Wang YB, Ding RR, Zhou XL, et al. Comparative evaluation of GPR versus APRI and FIB-4 in predicting different levels of liver fibrosis of chronic hepatitis B. Journal of Viral Hepatitis 2018; 25(5):581-589. 